La institucionalización de la planificación estratégica en la Universidad Nacional de La Plata

Rocío Tauber

Revista ES (en y sobre Educación Superior)

Vol.1, N¹-2 / Fecha de publicación: 27/12/2021

e-ISSN: $2718-6539$

https://revistas.unlp.edu.ar/ES/index

IIES - Facultad de Odontología

DOI: https://doi.org/10.24215/27186539e040

\title{
La institucionalización de la planificación estratégica en la Universidad Nacional de La Plata
}

The institutionalization of strategic planning at the National University of La Plata

A institucionalização do planejamento estratégico na Universidade Nacional de La Plata

Licenciada Rocío Tauber Instituto de Investigaciones en Educación Superior - UNLP https://orcid.org/0000-0002-0853-8230 rociotauber@hotmail.com 


\section{Resumen}

La Universidad Nacional de La Plata es pionera en planificación estratégica participativa. Se vuelve necesario para la gestión cotidiana como así también para los futuros escenarios, avanzar en la institucionalización formal de la tradición en planificación estratégica que viene teniendo nuestra Universidad desde hace 16 años. El presente Trabajo Final Integrador conforma y justifica los procedimientos que permitirán institucionalizar y formalizar el Plan de Desarrollo Institucional en una norma específica para que apruebe el Consejo Superior de la UNLP.

\section{Abstract}

The National University of La Plata is a pioneer in participatory strategic planning. It becomes necessary for day-to-day management as well as for future scenarios, to advance in the formal institutionalization of the tradition in strategic planning that our University has had for 16 years. This Integrative Final Work conforms and justifies the procedures that will allow to institutionalize and formalize the Institutional Development Plan in a specific norm to be approved by the Superior Council of the UNLP.

\section{Resumo}

A Universidade Nacional de La Plata é pioneira em planejamento estratégico participativo. Faz-se necessário, tanto para a gestão do dia a dia como para os cenários futuros, avançar na institucionalização formal da tradição de planejamento estratégico que nossa Universidade possui há 16 anos. Este Trabalho Final Integrativo conforma e justifica os procedimentos que permitirão 
institucionalizar e formalizar o Plano de Desenvolvimento Institucional em norma específica a ser aprovada pelo Conselho Superior da UNLP.

\section{Palabras clave}

UNLP, gestión, Plan de Desarrollo Institucional, Plan Estratégico, Planificación estratégica participativa.

\section{Keywords}

UNLP, management, Institutional Development Plan, Strategic Plan, participatory strategic planning.

\section{Palavras chave}

UNLP, gestão, Plano de Desenvolvimento Institucional, Plano Estratégico, planejamento estratégico participativo.

Las instituciones, para que puedan conducir los destinos de su comunidad, necesitan tener un Plan y contar con una estrategia para poder llevarlo a cabo. Es así que, para poder conducir primero tienen que ser planificadoras y estrategas. "Planificar estratégicamente permite que las tácticas coyunturales formen parte coherente del mejor camino hacia el futuro y que los Planes de Gestión formen parte de una Estrategia Integral de Desarrollo consensuada y sin actores excluyentes, con objetivos y prioridades claras y compartidas, definidas por aspiraciones, necesidades y posibilidades del conjunto social" (Tauber, 2004, pág. 16).

La UNLP conduce a su comunidad, cuenta con un Plan y tiene una Estrategia. Prueba de esto es que planifica estratégicamente desde el año 2004, 
convirtiéndose en pionera en el diseño e implementación de Planes Estratégicos en Universidades. La primera experiencia fue en el período 20042007, y luego le siguieron otras versiones: 2007-2010, 2010-2014, 2014-2018 y 2018-2022 (en ejecución).

El Plan Estratégico (PE) es un instrumento que en todo este tiempo demostró ser dinámico, ordenador, participativo, transparente y democrático. Como así también logró tener un consenso en la comunidad universitaria que lo legitima. Sin embargo, a pesar de que desde sus inicios esa legitimación siempre estuvo implícita y se dio de manera consensuada por los integrantes de la Universidad, muchos de los procesos que conforman la planificación estratégica participativa de la UNLP necesitan una legitimación formal.

\section{UNLP: un crecimiento planificado}

El Plan Estratégico 2018-2022, actualmente en gestión, comenzó a pensarse a comienzos del 2017 con la participación de la comunidad universitaria. Se realizaron reuniones con actores y funcionarios para discutir, repensar y plasmar las prioridades y futuras acciones que se iban a llevar a cabo en la gestión. Además, se desarrollaron talleres con participación abierta a la comunidad universitaria, ordenados según las estrategias del PE y con una lógica temática transversal. En una primera instancia fueron de Diagnóstico y luego se realizaron pensando específicamente en Propuestas de Transformación. Con la información relevada de las entrevistas, los encuentros y los talleres, se realizó un Diagnóstico con un FODA incluido para poder tener un estado de situación de cara a los próximos cuatro años de gobierno.

A esto se sumó el rastreo y puesta en común de las prioridades de gestión, acciones en las que se haría especialmente hincapié para concretar los 
principales objetivos. Con todo este bagaje informativo y de experiencia acumulada de los Planes Estratégicos anteriores, se delineó el Plan Estratégico 2018-2022 que se hizo público en mayo del 2018. “El PE 2018-2022 está centrado en el rendimiento académico, la mejora en los índices de egreso, y la inclusión a través de herramientas de educación formal alternativa" (La Palabra Universitaria, 2018).

Para comprender el dinamismo del Plan y la evolución que ha venido teniendo la UNLP, el actual Plan Estratégico (2018-2022) se organizó en un comienzo con un Objetivo General que define la visión compartida de cómo debe ser la UNLP, un Modelo de Desarrollo Institucional donde queda plasmada la misión que asume nuestra Universidad, pero ahora cuenta con 6 Estrategias: Enseñanza, Investigación y Transferencia, Extensión, Arte y Cultura, Relaciones Institucionales, y Administración y Gestión. Cuando fue lanzado, contaba con 25 Programas Generales, 85 Programas Específicos, 225 Subprogramas y 680 Subprogramas operativos, Proyectos y Obras.

Para el presidente Fernando Tauber "hacer público el Plan es una sana costumbre que tiene esta Universidad al inicio de cada gestión de gobierno; es transparentar y dejar claramente establecido de cara a la sociedad cuáles son sus objetivos y sus metas. Además es explicar cómo, con qué metodologías, con qué herramientas técnicas y con qué recursos y estructuras se busca alcanzar esas metas" (Portal web, 2018).

Para poder hacer ello también realiza Informes de Gestión donde toda la estructura de Secretarías y Dependencias del Rectorado de la UNLP brindan información de manera cotidiana acerca de lo que se está planificado en el PE y se está realizando día a día. Estos Informes, que en sus inicios fueron trimestrales, y en la actualidad se desarrollan de manera más dinámica, sirven 
tanto como insumo para los funcionarios de la gestión, como de instrumentos para seguir haciendo transparente el trabajo cotidiano de nuestra Universidad mostrándole a la sociedad lo que se viene haciendo diariamente, cómo se invierten los recursos y qué resultados se obtienen.

\section{Rüpü: el camino de nuestra Universidad}

En los Talleres participativos de Diagnóstico y de Propuestas de Transformación que se llevaron a cabo durante el año 2017 como etapa previa a la conformación del PE 2018-2022 surgió la necesidad entre varias personas de la comunidad universitaria de contar con un nuevo mecanismo que mejore la dinámica de informar sobre el Plan Estratégico.

Fue así que para el Plan actual, con el equipo de trabajo de la Jefatura de Gabinete junto con el Centro Superior para el Procesamiento de la Información (CESPI), se ideó un sistema de seguimiento, control y evaluación del Plan Estratégico. La nueva herramienta online llamada Rüpü (significa "rumbo", "camino" en Mapuche) apunta a optimizar la dinámica de circulación de la información, reordena la distribución de tareas, y posibilita un mejor seguimiento y control de su desarrollo cotidiano para el cumplimiento de los objetivos. De esta manera, el Plan Estratégico como instrumento de planificación se vuelve más flexible, útil y eficaz para la gestión; ya que se realizan revisiones periódicas y permiten posibles adecuaciones. En su lógica interna se pueden ordenar los niveles ya existentes en el PE (Programa General, Programa Específico, Subprograma, Subprograma Operativo/Proyecto/Obra) dentro de la Estrategia correspondiente, como así también tener una lógica transversal según las líneas prioritarias de la gestión. Será en el nivel más bajo (Subprograma Operativo/Proyecto/Obra) donde se 
cargarán las acciones cotidianas. Además, no sólo permite informar sobre la actividad que se viene realizando, sino que también sirve como una herramienta de planificación interna hacia adentro de las dependencias que están informando.

Esto es posible porque Rüpü permite cargar fechas de inicio y fin, actores involucrados, responsables, áreas, dependencias, porcentaje de realización; como así también la posibilidad de informar sobre recursos humanos, presupuesto, etc. Asimismo, la herramienta brinda la posibilidad de leer toda esa información a través diversas herramientas de lectura como Diagramas de Gantt, calendarios, y se pueden descargar informes con la información detallada de los Subprogramas Operativos, Proyectos y Obras del Plan Estratégico.

\section{¿Por qué institucionalizar el Plan?}

Una de las cuestiones vinculadas al Plan Estratégico que no se ha realizado, es la creación de un marco normativo que regule e institucionalice los procedimientos de seguimiento y gestión del PE y de la instrumentación de Rüpü. Esta propuesta define un modo de conformar y justificar los procedimientos que permitan institucionalizar y formalizar el Plan de Desarrollo Institucional (PDI) en una norma específica para que apruebe el Consejo Superior de la UNLP.

Dicha resolución debería contener la descripción de la estructura de lo que ya viene siendo el Plan Estratégico en nuestra Universidad en donde se incluya la Misión, la Visión, los Valores, las Estrategias, el Cronograma de Acción, el Equipo de Coordinación responsable, etc. Su desarrollo debiera completarse con la descripción de las funciones, la designación de los roles, la asignación 
de presupuesto, y el desarrollo de un Protocolo de Diseño, Seguimiento y Evaluación del PE. Asimismo, y en la misma lógica de recorrer los caminos formales para una correcta institucionalización, se debiera aprobar Rüpü como herramienta de seguimiento, control y evaluación del PE.

Todo esto conforma el Objetivo General de este trabajo: consolidar la continuidad del Plan de Desarrollo Institucional como una herramienta integral de la política institucional permanente en la Universidad Nacional de La Plata. El Plan Estratégico, desde su primera versión en el año 2004 hasta el día de hoy fue impulsado por su Director, Fernando Tauber, y se fue llevando adelante por decisión consensuada de la comunidad universitaria; pero nunca contó con la aprobación por parte del Consejo Superior, uno de los órganos de máxima autoridad de la UNLP.

El 13 de marzo del 2020 se publicó la Resolución N 635 firmada por el Presidente de la UNLP en la cual se aprobó el Plan Estratégico 2018-2022 y se encomienda a la Jefatura de Gabinete la coordinación general de todas las acciones de su implementación, seguimiento, evaluación y eventuales correcciones.

Asimismo también se especifica que estas acciones de coordinación de la Jefatura de Gabinete son con vinculación con el Instituto de Investigaciones en Educación Superior (IIES) y el Centro Superior de Procesamiento de la Información (CESPI), para trabajar en pos de la instrumentación del PE con dinámicas y herramientas que potencien su accesibilidad y funcionamiento tecnológico y procedimental (Resolución №635, 2020).

La reciente Resolución representa un punto muy positivo y alentador para la institucionalización de la planificación estratégica participativa de la cual la UNLP fue pionera, gestionando con Planes Estratégicos desde hace 16 años. 
Desde el 2004 hasta el 2020 ya cuenta con cinco versiones distintas (20042007, 2007-2010, 2010-2014, 2014-2018, 2018-2022) adecuadas a la coyuntura y adaptadas a las distintas prioridades, pero nunca había contado con una normativa que lo convirtiera en formalmente oficial siendo aprobado por el gobierno de la UNLP.

Sin embargo, la Resolución № 635 atiende puntualmente a la existencia del Plan Estratégico y la asignación de la Jefatura de Gabinete en su rol de coordinadora del mismo. En esta primera instancia apunta a algo más específico e instrumental.

Aún queda por fuera toda la cultura de la planificación estratégica que la contiene. Sigue siendo importante contar con una Resolución más abarcativa que describa su contenido, regule sus procedimientos, defina un equipo de coordinación, delimite roles y funciones, asigne presupuesto y recursos.

EI PE se ha venido gestionando a lo largo de todos estos años de manera regular, pero nunca contó con un presupuesto asignado para llevarlo a cabo, a pesar de que las tareas que se realizan son cotidianas e implican una inversión importante de recursos humanos, materiales y temporales.

Hasta la gestión pasada, donde el Lic. Raúl Perdomo era el Presidente de la UNLP, las acciones que se venían llevando a cabo sobre Plan Estratégico estaban siendo informadas de manera directa hacia el Director del PE a través de un documento en formato word donde cada área informaba de manera trimestral acerca de sus avances en las acciones. Esto resultaba tedioso tanto para quien informaba, como para quien debía leer la información que llegaba a tener una gran densidad.

A partir de la actual gestión y a la par del comienzo del Plan Estratégico 20182022, se incorporó el sistema online Rüpü que volvió mucho más dinámica, 
transversal, colectiva, eficiente y eficaz la información. Esta herramienta fue presentada y estuvo acompañada de reuniones y diversas capacitaciones al personal de la gestión para apropiarse de su uso, pero tampoco fue formalmente aprobada por el Consejo Superior, como el instrumento de seguimiento y control del Plan Estratégico de la UNLP.

El Plan Estratégico no es un simple instrumento técnico o un manual de procedimientos. EI PE es una herramienta para institucionalizar una estrategia de gestión a largo plazo, pero además hoy por hoy forma parte de algo mucho más abarcativo que contiene toda una lógica de tradición en planificación estratégica participativa que viene teniendo nuestra Universidad. $Y$ eso va mucho más allá del Plan en sí. Es por eso que se vuelve necesario formalizar procesos administrativos que garanticen su buen funcionamiento y los instalen definitivamente en el accionar de la gestión de la UNLP.

En este sentido, la propuesta del Proyecto de Intervención apunta a la importancia de institucionalizar formalmente y reafirmar en la práctica, la cultura de planificación estratégica que viene teniendo nuestra Universidad desde hace 16 años.

El producto final es el análisis propositivo que origine la norma del Consejo Superior en donde quede establecida la estructura que debe institucionalizar el Plan de Desarrollo Institucional, el área responsable, el equipo de trabajo a cargo con sus roles y funciones, las tareas específicas, y su procedimiento.

Dicho documento contiene:

- El "Pensar la Universidad", como un texto que representa al Proyecto Institucional de la UNLP para cada período de gobierno y en el cual quedan plasmadas las líneas prioritarias de gestión; 
- El Plan Estratégico, como instrumento que contiene las Estrategias, los Programas (Generales y Específicos) y los Subprogramas Operativos / Proyectos / Obras;

- Rüpü, como herramienta de seguimiento y control del Plan en curso y en donde está alojado todo el accionar cotidiano;

- Los Informes de Gestión, como los documentos donde la Universidad hace público su trabajo hacia adentro de la propia institución y hacia afuera de la comunidad universitaria; además de ser de gran utilidad para obtener información en tiempo y forma para la toma de decisiones de la gestión;

- Un Protocolo de Seguimiento, Control y Evaluación del Plan Estratégico, donde queden claros de manera general los procedimientos que se deben llevar a cabo para una gestión eficiente del Plan; y

- La Asignación de recursos económicos, materiales y humanos. Donde exista una asignación de presupuesto destinada a la planificación estratégica en la UNLP, como así también una distribución de materiales. En el mismo sentido, es fundamental crear un Equipo de Coordinación del Plan Estratégico que se encargue de gestionar estas tareas. Dicho equipo tiene que tener roles y funciones claramente designadas, y un lugar físico de trabajo. Asimismo, allí es donde se realizarán tareas de investigación para fortalecer y mejorar su operatividad.

Avanzar en la tarea de institucionalizar todo lo anteriormente mencionado será un objetivo necesario para seguir afirmando y garantizar el buen funcionamiento de la planificación estratégica participativa en la UNLP.

\section{¿Cuál es el resultado esperado?}


Atendiendo al objetivo general del Proyecto que es contar con una Resolución que englobe la totalidad del PDI de la UNLP, entre los resultados esperados es poder ampliar el rango de cobertura de la Resolución N 635 para que contenga todo lo vinculado a la planificación estratégica participativa. A pesar de que dicha resolución aprobó formalmente por primera vez un Plan Estratégico en la UNLP, aún queda consolidar otras tantas aristas que tienen que ver con la planificación estratégica participativa en la UNLP hoy.

En esa misma dirección, la Resolución N 635 además asignó a la Jefatura de Gabinete como el área que se encargará de la coordinación del PE en vinculación específica con el IIES. Es cierto que en los últimos años se está trabajando bajo las directivas del Jefe de Gabinete de la UNLP desarrollando tareas técnicas y operativas vinculadas al PE, al Rüpü, al desarrollo de Informes (de gestión, de líneas estratégicas, de análisis), a las actividades de Evaluación Institucional, entre otras. No obstante, sería importante conformar formalmente un Equipo de Coordinación del PDI con área de desempeño en el IIES/Jefatura de Gabinete que contenga funciones y roles claramente definidos para darle un marco de legalidad que clarifique su conformación y lo haga perdurable en el tiempo. En consecuencia, otro de los resultados esperados tiene que ver con institucionalizar un equipo/espacio de trabajo vinculado específicamente al PDI, para visibilizar aún más el trabajo que se viene realizando y que toda la comunidad, hacia adentro y hacia afuera de la UNLP, tengan conocimiento sobre ello.

En lo que respecta a protocolizar los pasos a seguir para el desarrollo del PDI, lo que se espera es conformar un ordenamiento que simplifique el accionar cotidiano, como así también la conformación de los futuros Proyectos Institucionales, Planes Estratégicos, desarrollo de informes, etc. 
Asimismo, también se espera descentralizar la dirección del Plan Estratégico de la figura de su Director (que en la actualidad es el Presidente de la UNLP) para aliviar las tareas que ello implica y realizar un trabajo colaborativo. Desde su creación en el 2004, el PE estuvo dirigido por el Dr. Fernando Tauber y desde aquel año hasta el PE 2014-2018 los trabajos de dirección, control, seguimiento y realización de informes estuvieron centrados solamente en su persona. Esto se trata de un trabajo muy arduo que implica mucho tiempo de dedicación.

Paulatinamente, con el actual PE (2018-2022) comenzó a cambiar con una participación muy activa y fuerte por parte del Jefe de Gabinete y de su equipo de trabajo. Sin embargo, aún resta delegar de manera ordenada y planificada más actividades vinculadas al PE y al PDI en general. En este sentido, despersonalizar su gestión es otro de los resultados esperados.

\section{Reflexiones Finales: Sigamos eligiendo el futuro, sigamos planificando}

En el año 2004 se produjo uno de los hitos en las discusiones sobre educación superior en nuestro país. En Horco Molle, Provincia de Tucumán, se realizaron las Primeras Jornadas de Reflexión sobre Educación Superior en la Argentina, donde los Rectores miembros del Consejo Interuniversitario Nacional acordaron avanzar en acciones sobre el futuro de la universidad argentina vinculadas a los objetivos de la formación de ciudadanos libres, comprometidos con la sociedad democrática, con la calidad y diversidad de saberes científicos, técnicos, humanísticos, y culturales; entre otros compromisos (CIN, 2004).

En este sentido, "actualizar y consolidar los proyectos institucionales de cada Universidad como una herramienta para su desarrollo" (CIN, 2004) aparece como una de la acciones más relevantes que se acordaron en consonancia con 
una visión contemporánea y comprometida de la misión de la Universidad Argentina.

Asimismo, otro de los documentos relevantes es la Declaración de La Plata en el año del Bicentenario, de octubre del 2010. En el marco del CIN, los rectores de las universidades públicas argentinas reflexionaron y realizaron aportes significativos sobre las demandas y desafíos de la educación superior argentina contemporánea. Entre los puntos concluyentes se destaca el de "avanzar en la planificación estratégica y participativa, la administración transparente y la gestión eficaz del desarrollo institucional con criterios de eficiencia, eficacia, comunicabilidad, flexibilidad y trabajo en equipo".

Estos son sólo dos de muchos acontecimientos y documentos que en la discusión profunda sobre las universidades públicas en Argentina, coinciden en que la planificación estratégica institucional es una herramienta indispensable para el desarrollo de las instituciones de educación superior.

Con orgullo se debe decir que la UNLP viene haciendo esto hace años y ya ha construido su propia historia sobre ello: desde el PE 2004-2007 hasta el que está actualmente en gestión; los sucesivos "Pensar la Universidad" que plasmaban los Proyectos Institucionales de cada período de gobierno universitario y sus líneas prioritarias de gestión; los organigramas institucionales que fueron variando a lo largo del tiempo, reflejando las demandas de la sociedad y los objetivos de la Universidad; la creación de Rüpü como herramienta de seguimiento y control de las acciones que responden a la planificación estratégica y que significó un gran salto mejorando la evaluación, los ajustes y replanteos, como así también en la realización de informes que sirven para hacer foco en la gestión cotidiana; la decisión de iniciar nuevamente un proceso de Evaluación Institucional, para poder autoevaluarnos 
y asimismo, decidir que nos miren desde afuera con la profunda intención de seguir mejorando día a día; y hasta la reciente Resolución $N^{\circ} 635$ que no sólo aprueba el PE 2018-2022 sino que decide que la Jefatura de Gabinete de la UNLP sea la coordinadora. Todo esto resumido muy genéricamente contiene el Plan de Desarrollo Institucional de la UNLP. Validado por los actores de la comunidad universitaria, el PDI es el instrumento ordenador de la gestión y de su rumbo.

No obstante, aquí también entra en juego la importancia de la legitimación porque un PDI sin la aprobación y el consentimiento de sus actores internos y externos carecería de sentido. Tanto los funcionarios de la gestión como todo el resto de la comunidad universitaria deben apropiarse de la planificación estratégica de la Universidad de la que forman parte; pero también la sociedad en general tiene que tener acceso a saber sobre lo que se está haciendo y sobre lo que se quiere hacer en la institución.

Esto tiene que ver con algo que es central para nuestra UNLP que es la búsqueda de consensos. En el Informe Narrativo de Autoevaluación de la UNLP que fue entregado para el último proceso de Evaluación Institucional a la CONEAU, se destaca que como comunidad hemos aprendido que, a pesar de que es un camino difícil y que lleva mucho esfuerzo, el consenso es la base más fuerte para construir con solidez los cimientos para sostener con fuerza el desarrollo de nuestra Universidad, una Universidad más equitativa para el conjunto de la sociedad (Universidad Nacional de La Plata, 2019).

Aún queda mucho por recorrer en materia de planificación estratégica en la UNLP para hacer más eficiente y eficaz la gestión. Un camino que debemos comenzar a transitar es el de delinear una serie de indicadores que permitan poder evaluar lo que se está haciendo en torno al PE, y así poder ajustar y 
replantear lo que sea necesario para lograr los objetivos deseados. Esta será una tarea ardua que debemos llevar adelante como equipo de trabajo junto con el Jefe de Gabinete, el Presidente y todos los responsables de las áreas de Presidencia.

La UNLP es una institución inmensa, variada y muy compleja. Siempre va a haber mucho por hacer, por eso se vuelve central tener un Proyector de Desarrollo Institucional que contenga toda esa enormidad, con un Plan Estratégico a la cabeza que lo lleve adelante e incluya objetivos claros y diga cómo alcanzarlos, donde se gestionen las prioridades y se ilumine el rumbo. Mientras tanto continuamos trabajando, seguimos construyendo futuro, seguimos planificando.

\section{Bibliografía}

- Bonicatto, M. (2017). Gestión estratégica planificada (GEP): un método para la gestión en organizaciones públicas. La Plata, Buenos Aires, Argentina: EDULP.

- Bonicatto, M. (2019). Impreparados para gobernar. ¿La Planificación y la Gestión Estratégica son posibles? Documento inédito. La Plata, Buenos Aires, Argentina.

- Casareto, L. M., Aragón, M. M., \& Panella, C. (2018). Guía documental y bibliográfica: de la Universidad Provincial a la reforma Universitaria en La Plata 1890-1921. Recuperado el 20 de febrero de 2020, de SEDICI: http://sedici.unlp.edu.ar/bitstream/handle/10915/68737/Documento_com pleto.pdf-PDFA.pdf?sequence=1\&isAllowed=y

- CIN (Consejo Interuniversitario Nacional). (27 de agosto de 2004). Declaración de Horco Molle. Primeras Jornadas de Reflexión sobre la 
Educación Superior en la Argentina. Plenario del Consejo Interuniversitario Nacional. Tucumán, Argentina.

- CIN (Consejo Interuniversitario Nacional). (2010). Las universidades públicas en el año del bicentenario. Declaración de La Plata. La Plata, Buenos Aires, Argentina.

- Clark, R. I. (2017). Capítulo II. Problemática jurídica de la autonomía universitaria. En R. I. Clark, Autonomía universitaria y control judicial (págs. 49-77). La Plata: Ad-Hoc.

- Clark, R. I. (2017). Capítulo III. Problemática sustancial del control judicial sobre las universidades. En R. I. Clark, Autonomía universitaria y control judicial (págs. 79-113). La Plata: Had-Hoc.

- Conci, M. C., Moine, M. B., \& Tamagno, M. R. (2016). La aplicación de la planificación estratégica en instituciones universitarias. Villa María: Universidad Nacional de Villa María.

- Estatuto de la Universidad Nacional de La Plata. (2008). La Plata, Buenos Aires, Argentina.

- Giordano, C. J. (2009). 23 tesis sobre la tesis. La comunicación para la transformación de la gestión educativa (Tesis de Doctorado). La Plata, Buenos Aires, Argentina: Universidad Nacional de La Plata.

- Huerta, F. (1993). El Método PES. Fundación Altadir.

- Illescas, N. (2017). Análisis del Planeamiento en las Universidades Nacionales en la Argentina. El caso del Plan de Desarrollo Institucional 2010-2019 de la Universidad Nacional del Litoral (Tesis de Maestría). Santa Fe: Universidad Nacional del Litoral.

- Matus, C. (1987). Política, planificación y gobierno. Buenos Aires: Fundación Altadir. 
- Osorio, A. (2003). Planeamiento estratégico. Buenos Aires: INAP.

- Porta, P. I. (2014). Comunicación del conocimiento/Conocimiento de la Comunicación (Tesis de Doctorado). La Plata: Universidad Nacional de La Plata.

- Tauber, F., Pintos, P., Longo, J., Delucchi, D., \& Martino, H. (2002). Procesos de innovación en la gestión local: Planificación Estratégica en tiempos de crisis. La necesidad de la permanente readecuación metodológica. IV ${ }^{\circ}$ Seminario Nacional de REDMUNI. Córdoba, Argentina.

- Tauber, F. (2004). Modulo VII. Planeación Institucional (Planeamiento Estratégico y Continuo). Especialización de Postgrado en Liderazgo Universitario. Managua, Nicaragua: Universidad Nacional de La Plata.

- Tauber, F., Delucchi, D., Martino, H., \& Pintos, P. (2006). La Planificación Estratégica Participativa. Para el desarrollo urbano y regional. La Plata: Edulp.

- Tauber, F. (2009). Comunicación en la planificación y gestión de las universidades públicas argentinas: el caso de la UNLP en el trienio junio 2004-mayo 2007 (Tesis de Doctorado). La Plata: Universidad Nacional de La Plata.

- Tauber, F. (2015). Hacia el segundo manifiesto: los estudiantes universitarios. La Plata: EDULP.

- Tauber, F. (2016). Pensar la Universidad. Proyecto Institucional de la Universidad Nacional de La Plata 2018-2022. La Plata, Buenos Aires: Publicación Institucional UNLP. 
- Universidad Nacional de La Plata. (2004). Plan Estratégico 2004-2007. Recuperado el 1 de marzo de 2020, de Portal web UNLP: https://unlp.edu.ar/

- Universidad Nacional de La Plata. (2007). Plan Estratégico 2007-2010. Recuperado el 1 de marzo de 2020, de Portal web UNLP: https://unlp.edu.ar/

- Universidad Nacional de La Plata. (2010). Plan Estratégico 2010-2014. Recuperado el 1 de marzo de 2020, de Portal web UNLP: https://unlp.edu.ar/

- Universidad Nacional de La Plata. (2014). Plan Estratégico 2014-2018. Recuperado el 1 de marzo de 2020, de Portal web UNLP: https://unlp.edu.ar/

- Universidad Nacional de La Plata. (2018). Plan Estratégico 2018-2022. Recuperado el 7 de junio de 2019, de Portal web UNLP: https://unlp.edu.ar

- Universidad Nacional de La Plata. (2019). Informe Narrativo Autoevaluación CONEAU. La Plata: UNLP.

- Universidad Nacional de La Plata. (2019). Organigrama Institucional. La Plata, Buenos Aires, Argentina.

- Vega, R. I. (7-9 de mayo de 2003). El planeamiento estratégico en la universidad argentina: aspectos problemáticos. III Coloquio Internacional sobre Gestión Universitaria de América del Sur. Buenos Aires, Argentina.

- Vega, R. I. (2005). Desafíos de la gestión universitaria. En M. Efrón, \& R. I. Vega, Aportes al debate sobre la gestión universitaria II (págs. 236256). Buenos Aires: De los cuatro vientos editorial. 
- Vega, R. I. (2012). Coloquios de Gestión Universitaria de América del Sur: el por qué y el cómo de su surgimiento. En N. Fernández Lamarra, La gestión universitaria en América Latina (págs. 71-91). Coronel Oviedo, Paraguay: Universidad Nacional del Caaguazú. 